\title{
Symbolic Indexing of Cardiological Sequences through Dynamic Curve Representations
}

\author{
M.Baroni ${ }^{1}$, G.Congiu ${ }^{2}$, A.Del Bimbo ${ }^{2,3}$, A.Evangelisti ${ }^{2}$, and E.Vicario ${ }^{2}$ \\ 1 Dip. Ingegneria Elettronica, Università di Firenze, Italy \\ 2 Dip. Sistemi e Informatica, Università di Firenze, Italy \\ 3 Dip. Elettronica per l'Automazione, Università di Brescia, Italy
}

\begin{abstract}
Digital image analysis supports diagnostic activities by highlighting geometric and temporal features of physiological phenomena that are not perceivable to the human observation. These features can be exploited to build up symbolic representations of visual data in medical reports and to index them within large databases. The comparison of such representations against descriptive queries capturing the properties of significant physiological phenomena supports new diagnostic approaches through the systematic analysis of database reports.

A prototype system is presented which supports the construction of symbolic representations and their comparison against descriptive queries capturing geometric and temporal properties of time-varying $2 \mathrm{D}$ shapes deriving from dynamic cardiac analyses. The system is embedded within a visual shell allowing physicians to compose content-oriented queries through iconic interaction.
\end{abstract}

\section{Introduction}

Imaging processing and image databases are assuming a growing relevance in medical systems supporting physicians in diagnostic activities through an objective and reproducible exploitation of their experience. Digital image processing and analysis permit the observation of geometric and temporal features of physiological phenomena that are not evident, or even not perceivable, for human observation. These features can be exploited to build up symbolic representations of visual data in medical reports and to index them within large databases. The comparison of symbolic representations against descriptive queries capturing the properties of significant phenomena supports content-oriented analysis of the reports stored in a database and opens the way to systematic diagnostic approaches. On the one hand, in prospective diagnosis, a number of standard queries are checked against the indexes of a single report to accomplish a screening of the possible pathologies of a specific patient. On the other hand, in retrospective diagnosis, a single query is checked against a large number of reports to identify pathological classes and to investigate them against historical data.

A number of indexing techniques have been proposed which can be exploited to support the symbolic representation of the contents of single static images [7] [4]. But, only a few description techniques have been recently proposed, which can be exploited in the representation of multiple sequenced images [10] [6]. None 
of these techniques addresses the case of non-rigid imaged shapes, as encountered in most medical applications.

In this paper, a prototype system is presented which supports the construction of symbolic representations and their comparison against descriptive queries for time varying $2 \mathrm{D}$ shapes deriving from dynamic cardiac analysis ${ }^{4}$. Qualitative static and dynamic shape descriptors are introduced to provide a symbolic representation of shapes, and a descriptive language is presented which supports the expression of queries on their contents. System interaction is accomplished through a visual language allowing physicians to compose iconic queries on geometric and temporal properties of reports to be retrieved.

\section{Visual Data Acquisition and Processing}

Cineangiographic analysis produces image sequences presenting the evolution over a complete beating period of the contour of the left ventricle ( $\mathrm{LV}$ ) in the Right Anterior Oblique projection of the heart. Image acquisition is performed on $35 \mathrm{~mm}$ films at a rate of 50 frames/second during cardiac catheterization, in mild inspiration. LV contours are traced and digitized frame-to-frame, from the beginning to the end of one cardiac cycle (according to ECG signal), by using a moviola and a graphic tablet (MY-PAD-A3 digitizer, model K-510mk2, $0.1 \mathrm{~mm}$ resolution).

At storage time, each cineangiographic report is given as a sequence of sample curves represented in a chain code numerical format. This representation is used to extract the bending function of the individual samples [2], which are then used to build the symbolic representation of the contents of the report according to the description format expounded in Sect.3. The numerical chain code representation of the report is stored in the system along with this symbolic index.

The resulting contour of each frame is processed to derive the normalized curvature function. This comprises an invariant description with respect to rigid transformations and provides local information independent of geometrical assumptions. Curvature values are also correlated with wall stress and evidence local shape characteristics, such as concavity, protrusions and flat segments. To reduce quantization and statistical errors, the curvature function is computed through an adaptive Fourier approximation. The contour is first approximated by a windowed 2D Fourier series which is then differentiated to obtain curvature values in close form. Approximation errors and curvature smoothness are optimized by changing the harmonic number and filter-window along the contour. The benefits of this approach are discussed in [2] with comparison to other commonly used methods. The interobserver and intraobserver repeatability of the entire procedure, from the contour digitalization to the curvature computation, accomplishes a mean deviation lower than $10 \%$ and a linear correlation coefficient higher than $90 \%$.

4 The system was developed in cooperation with the Institute of Cardiology of the University of Florence, Italy, and it presently runs on an IBM Risc R6000. 


\section{Visual Data Representation}

Following the results of studies on human perception, the representation of the shape of a curve can be reduced to the representation of a set of sub-curves, each characterized by homogeneous features [11]. Different partitioning criteria have been proposed in the literature to identify subcurves [8] [9]. In particular, in the codon approach [12], a curve is divided in subcurves, the codons, which are delimited by two subsequent minima of the bending function. This segmentation approach appears suited to cineangiographic reports as it reflects a natural partitionment of the heart body into physiological regions characterized by different values of internal tension forces. This segmentation also prescinds from both the orientation and the zooming factor employed during the imaging process.

Following this approach, each frame of the ventricular contour is represented through a (variable) number of codons, each following one out of 5 possible patterns corresponding to the different combinations of the signs of the bending function in the extrema and by the possible presence of intermediate flexes (see Fig.1). Codons are also associated with three symbolic attributes of symmetry, bending, and extension, accounting for the position of the bending maximum with respect to the codon center, the maximum value of the bending function along the codon, and the linear distance between the extrema of the codon. All the attributes are expressed in qualitative terms.
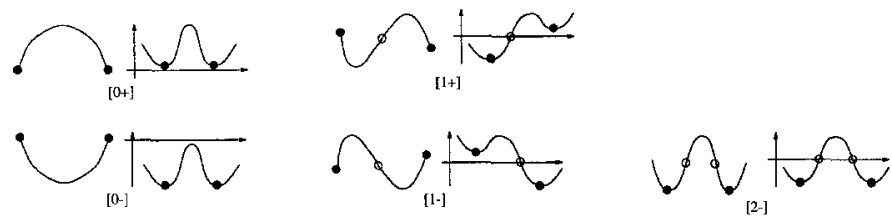

Fig. 1. Codon types corresponding to the five possible combinations of the sign of bending minima and of the number of flex points between the extrema.

The evolution over time of the curve is captured by associating codons belonging to subsequent frames through a tracking relation. In the common case, a single codon of sample $n$ is biunivocally associated with a single codon of sample $n+1$, however, due to the appearance or disappearance of bending minima, it may also be the case that a codon of sample $n$ is mapped onto multiple codons of sample $n+1$ or that multiple codons of sample $n$ are mapped onto a single codon of sample $n+1$. These merging and splitting phenomena, which are quite frequent in cineangiographic contours, are resolved through an heuristic decision which associates codons in subsequent samples on a minimum displacement basis [5]. An example of tracking is reported in Fig.2.

In general, the tracking relation identifies three disjoint sets of codons, which are never intertwined by mutual splitting or merging. This is a general property of cineangiographic reports, and reflects the fact that the heart is naturally 
divided into three different anatomical regions, that are usually referred to as anterior, apical and inferior. This result permits the automatic attribution of each part of each sample of the curve to one of these three anatomical regions.

The comparison of subtended areas of codons that are associated to each other by the tracking permits a symbolic characterization of the expansion/contraction trend, which is mainly relevant to the purposes of the diagnostic activity. To capture this trend, the individual codons of each cineangiographic sample are associated with a further attribute of straining which is interpreted as expansive, stable or contractive. The straining attribute provides a local representation of the evolution of the curve in that it refers to the individual codons. The simultaneous presence of strongly differentiated straining trends is a condition of main relevance as it is indicative of important pathologies.

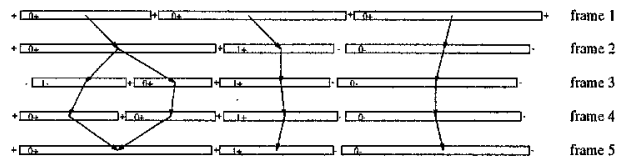

Fig. 2. Codon tracking for six samples of a cineangiographic report. For each sample, the curve is represented in curvilinear abscissa and it is segmented into codons that are tracked along subsequent samples. The first codon of the first sample is tracked onto the first codon of the second sample. In turn, this is split into the first and second codon of the third sample. The first and second codon of the fourth sample are merged into the first codon of the fifth sample.

\section{Sequence Querying by Contents}

In the retrieval stage, the user expresses content-oriented queries through a visual iconic interface by defining distinctive geometric conditions which characterize sequences to be retrieved and temporal ordering relationships among the lasting intervals of these conditions. Visual queries are automatically interpreted by the system and checked against the descriptions of the sequences in the database.

The basic graphic objects employed in the visual expression of queries are icons and lines. Icons represent significant conditions, such as an expansive straining or the presence of a sharp bending in some of the codons of the cineangiographic curve. Multiple icons can be grouped visually to express Boolean composition of multiple events. Lines denote the lasting time intervals in which conditions and condition compositions occur, and they are annotated with the iconic representation of the condition composition. Each line is also annotated with a special icon denoting the anatomical region in which the event composition occurs and the reference phases of systolic contraction and diastolic expansion. 
The mutual positioninig of event lines on the screen defines the temporal ordering among the lasting intervals of distinctive events. To permit the expression of don't care conditions in the interpretation of this mutual positioning, each line is associated with one or more colors, and the mutual position between intervals which do not share any common color is ignored in the interpretation of the visual arrangement. The translation of the visual specification occurs through two distinct phases. First, the mutual positioning of event intervals are analyzed and classified into one of the 13 possible mutual ordering relations [1]. Afterwards, icon compositions associated with each interval are parsed and translated into interval formulae. These formulae are checked against the descriptions of the sequences in the database, and matching reports are displayed on the screen.
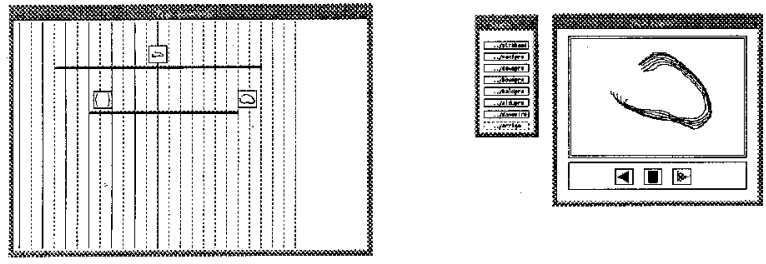

Fig. 3. Fig a: a visual query expressing a contractive strain in the apical region during the systolic phase. Fig b: the list of retrieved reports(left hand side menu) and display (in the right hand side window) frame by frame of one of them.

Fig.3a shows a visual query seeking for reports characterized by an inward motion in the apical region during the diastolic phase. The upper line is annotated with the systolic icon thus denoting the time interval in which the heart should expand. The lower line is annotated with two icons representing a contractive strain (in the middle of the line) and the apical region (in the right hand side of the line) so as to represent a time interval in which part of the apical region exhibit a contractive motion. The two lines are associated with a common color so as to make significant the mutual positioning of the two intervals. The system interpretation of the overall arrangement produces a query statement which causes the retrieval of the reports characterized by a time interval which is contained in the systolic phase and which exhibits a contractive motion in the apical region. When retrieval is completed, reference names of retrieved reports are listed on a menu which permits selection and display on the screen of reports of specific interest for the user (Fig.3ab).

Fig.4a shows a refinement of the query of Fig.3, which adds the request for an outward motion in the apical region during the systolic phase. Two further lines are added (in the bottom part of the screen), one standing for the systolic phase and the other for the lasting interval of the expansive motion in the apical region. The two lines are associated with a common color different from that associating the previous two lines. As a result, the system takes care of the 
ordering between the two new lines and between the two old lines, but not of the the ordering between new and old lines. Again, after the interpretation of the query, retrieval is carried out and matching reports are listed and displayed on the screen (see Fig.4b).
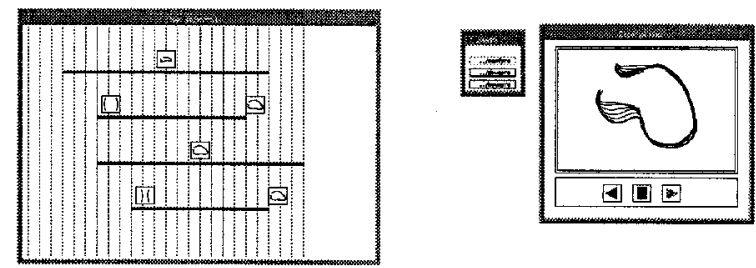

Fig.4. A refinement of the query of Fig.3 and the corresponing result.

\section{References}

1. J.F. Allen, "Maintaining Knowledge about Temporal Intervals," in Comunications of the ACM, Vol.26, n.11, Nov. 1983.

2. M.Baroni,G.Barletta, "Digital Curvature Estimation for Left Ventricular Shape Analysis," Image and Vision Computing, 1992.

3. E.Binaghi, I.Gagliardi, R.Schettini, "Indexing and Fuzzy Logic-Based Retrieval of Color Images," in IFIP Trans. Visual Database Systems II, Knuth, Wegner (Eds.), Elsevier Pub. 1992.

4. S.K.Chang, Q.Y.Shi, C.W.Yan, "Iconic Indexing by 2-D Strings", IEEE Transactions on Pattern Analysis and Machine Intelligence, Vol.9, No.3, July 1987.

5. G.Congiu, "Rappresentazione e Ricerca Visuale di Sequenze di Immagini Cardiologiche (In Italian)," Doctoral Thesis, Univ. Florence, Italy, July, 1994.

6. A.Del Bimbo, E.Vicario, D.Zingoni, "Symbolic Description and Visual querying of Image Sequences Using Spatio-Temporal Logic," accepted for publication on IEEE Transactions on Knowledge and Data Engineering.

7. K.Hirata, T.Kato, "Query by Visual Example: Content-Based Image Retrieval," In Advances in Database Technology - EDBT'92, A.Pirotte, C.Delobel, G.Gottlob (Eds.), Lecture Notes on Computer Science, Vol.580, Springer Verlag, Berlin, 1992.

8. M.Leyton, "Shape and Casual History," in Visual Form, Plenum Press, 1982.

9. S.Marshall, "Review of Shape Coding Techniques," in Image and Vision Computing, Nov.1989.

10. A.Nagasaka, Y.Tanaka, "Automatic Video Indexing and Full Video Search for Object Appearances," in IFIP Trans. Visual Database Sys. II, Knuth, Wegner (Eds.), Elsevier Pub. 1992.

11. S.E.Palmer, "The Psychology of Perceptual Organization,: a Transformational Approach," in Human and Machine Vision, Academic Press, New York, 1983.

12. W.Richards, D.D.Hoffman, "Codon Constraints on Closed 2D Shapes," in Computer Vision II, Natural Computation Group, M.I.T., Caribridge, 1984. 